University of Wollongong

Research Online

Faculty of Engineering and Information

Faculty of Engineering and Information

Sciences - Papers: Part B

Sciences

2020

\title{
MRI-LINAC beam profile measurements using a plastic scintillation dosimeter.
}

Levi Madden

University of Wollongong, ljm898@uowmail.edu.au

James Archer

University of Wollongong, jia335@uowmail.edu.au

Enbang Li

University of Wollongong, enbang@uow.edu.au

Urszula Jelen

Bin Dong

See next page for additional authors

Follow this and additional works at: https://ro.uow.edu.au/eispapers1

Part of the Engineering Commons, and the Science and Technology Studies Commons

Research Online is the open access institutional repository for the University of Wollongong. For further information contact the UOW Library: research-pubs@uow.edu.au 


\title{
MRI-LINAC beam profile measurements using a plastic scintillation dosimeter.
}

\begin{abstract}
(C) 2020 Associazione Italiana di Fisica Medica Plastic scintillation dosimeters (PSDs) possess many desirable qualities for dosimetry with LINACs. These qualities are expected to make PSDs effective for MRI-LINAC dosimetry, however little research has been conducted investigating their dosimetric performance with MRI-LINACs. In this work, an in-house PSD was used to measure 8 beam profiles with an in-line MRI-LINAC, compared with film measurements. One dimensional global gamma indices $(\gamma)$ and corresponding $Y$ pass rates were calculated to compare PSD and film profiles for the $1 \% / 1 \mathrm{~mm}, 2 \% / 2 \mathrm{~mm}$ and $3 \% / 3 \mathrm{~mm}$ criterion. The mean global pass rates were $85.8 \%, 97.5 \%$ and $99.4 \%$ for the $1 \% / 1 \mathrm{~mm}, 2 \% / 2$ $\mathrm{mm}$ and $3 \% / 3 \mathrm{~mm}$ criteria, respectively. The majority of the $\gamma$ failures occurred in the penumbral regions. Penumbra widths were measured to be slightly narrower with the PSD compared to film, however, the uncertainties in the measured penumbra widths brought the PSD and film penumbra widths into agreement. Differences in dose were calculated between the PSD and film, and remained within $2.2 \%$ global agreement for the central regions and $1.5 \%$ global agreement for out of field regions. These values for range of agreement were similar to the those reported in the literature for other dosimeters which are trusted for relative MRI-LINAC dosimetry.
\end{abstract}

\section{Disciplines}

Engineering | Science and Technology Studies

\section{Publication Details}

Madden, L., Archer, J., Li, E., Jelen, U., Dong, B., Holloway, L. \& Rozenfeld, A. (2020). MRI-LINAC beam profile measurements using a plastic scintillation dosimeter.. Physica Medica, 73 111-116.

\section{Authors}

Levi Madden, James Archer, Enbang Li, Urszula Jelen, Bin Dong, Lois C. Holloway, and Anatoly B. Rosenfeld 


\title{
MRI-LINAC beam profile measurements using a plastic scintillation dosimeter.
}

\author{
Levi Madden ${ }^{\mathrm{a}, \mathrm{b}}$, James Archer ${ }^{\mathrm{a}}$, Enbang Li ${ }^{\mathrm{a}, *}$, Urszula Jelen ${ }^{\mathrm{b}}$, Bin Dong ${ }^{\mathrm{b}}$, \\ Lois Holloway ${ }^{\mathrm{b}}$, Anatoly Rosenfeld ${ }^{\mathrm{a}, \mathrm{c}}$ \\ ${ }^{a}$ Centre for Medical Radiation Physics, University of Wollongong, NSW 2522, Australia \\ ${ }^{b}$ Ingham Institute for Applied Medical Research, Liverpool, NSW 2170, Australia \\ ${ }^{c}$ Illawarra Medical and Health Research Institute, University of Wollongong, NSW 2522, \\ Australia
}

\begin{abstract}
Plastic scintillation dosimeters (PSDs) possess many desirable qualities for dosimetry with LINACs. These qualities are expected to make PSDs effective for MRI-LINAC dosimetry, however little research has been conducted investigating their dosimetric performance with MRI-LINACs. In this work, an in-house PSD was used to measure 8 beam profiles with an in-line MRI-LINAC, compared with film measurements. One dimensional global gamma indices $(\gamma)$ and corresponding $\gamma$ pass rates were calculated to compare PSD and film profiles for the $1 \% / 1 \mathrm{~mm}, 2 \% / 2 \mathrm{~mm}$ and $3 \% / 3 \mathrm{~mm}$ criterion. The mean global pass rates were $85.8 \%, 97.5 \%$ and $99.4 \%$ for the $1 \% / 1 \mathrm{~mm}, 2 \% / 2 \mathrm{~mm}$ and $3 \% / 3 \mathrm{~mm}$ criteria, respectively. The majority of the $\gamma$ failures occurred in the penumbral regions. Penumbra widths were measured to be slightly narrower with the PSD compared to film, however, the uncertainties in the measured penumbra widths brought the PSD and film penumbra widths into agreement. Differences in dose were calculated between the PSD and film, and remained within $2.2 \%$ global agreement for the central regions and $1.5 \%$ global agreement for out of field regions. These values for range of agreement were similar to the those reported in the literature for other dosimeters which are trusted for relative MRI-LINAC dosimetry.
\end{abstract}

Keywords: MRI-LINAC, Fibre optic dosimeter, Plastic scintillator, dosimetry

\section{Introduction}

MRI-LINACs combine an MRI with a LINAC, where the MRI enables imaging of the patients with optimal soft tissue contrast while the LINAC delivers radiation therapy [1]. MRI-LINACs present a unique opportunity to achieve

\footnotetext{
* Corresponding author

Email address: enbang@uow.edu.au (Enbang Li )
} 
real-time adaptive radiation therapy and reduce the margins of treatment volumes [2]. However, the MRI's magnetic field influences the dose distributions delivered, alters a dosimeters response and its effects can increase response uncertainties [3. Many dosimeters have been investigated for relative dosimetry with MRI-LINACs including ionisation chambers, film dosimeters and solid state dosimeters. For MRI-LINAC dosimetry, an ionisation chambers response is dependent upon their orientation in the magnetic and radiation fields [3]. These dependences are correctable, however uncertainties can increase if deviations in chamber orientation occur [3]. Film dosimeters are water equivalent, have unmatched spatial resolution for 2D dosimetry and remain accurate for dosimetry with MRI-LINACs 4, 5, however they exhibit small changes to their dose response curves in the presence of magnetic fields [5]. Many solid state dosimeters are water equivalent, however, their responses are often dependent on their irradiation angle, which can inflate their uncertainties when applied for LINAC and MRI-LINAC dosimetry at varying irradiation angles [6, 7].

Similar to the aforementioned dosimeters, plastic scintillation dosimeters (PSDs) typically possess many of the desirable qualities for MRI-LINAC dosimetry without disadvantages associated with magnetic fields. These qualities include water equivalence for photons and electrons over the MRI-LINACs therapeutic energy range [8, 9, non-ferromagnetic composition and a real-time, linear response with dose 8. Additionally, plastic scintillators typically have irradiation angle independence [10, dose rate independence [10] and a response not degraded by magnetic fields [11].

PSDs are comprised of a plastic scintillator coupled to an optical fibre 12. An issue when using PSDs with LINACs arises with the generation of Cerenkov radiation in the PSD's optical fibre, occurring as a stem signal for PSDs [12. Cerenkov radiation is generated when an electron traverses through an optical medium at a speed greater than the local speed of light in that medium [13. The gold standard method for Cerenkov radiation correction, known as background subtraction [12, uses an optical fibre with no scintillator (referred to as a reference probe) to measure Cerenkov radiation only. In the background subtraction method, the PSD and reference probe are placed against each other and aligned to ensure that matching irradiation conditions are present between the PSD and reference probe. Given that matching optical fibres produce equal magnitudes of Cerenkov radiation for matching irradiation conditions, the PSD signal is corrected through subtraction of the reference probe signal.

In previous work [14, an in-house PSD was compared with a Farmer type ionisation chamber and a diamond detector for the measurement of output factors and a percent depth dose distribution. Disagreements between the three detectors were reported in the work and were attributed to their significantly different dimensions and sensitive volume geometries. In current work, we present MRI-LINAC beam profiles measured with an in-house PSD and compare these profiles with those measured by radiochromic film, considered the gold standard for beam profile measurements with an MRI-LINAC. 


\section{Materials and Methods}

The Australian MRI-LINAC uses an in-line setup, consisting of a $1 \mathrm{~T}$ open bore magnet (Agilent, UK) and a 6 MV Linatron-MP LINAC (Varex, USA) fitted with a Millennium 120 multi-leaf collimator (MLC)(Varian, USA). The MRI-LINAC is setup with the photon beam aligned parallel to the magnetic field (referred to as an in-line setup). The Australian MRI-LINAC uses a fixed horizontal beam line, being mounted on rails to allow for changes in sourceisocentre distance (SIDs). A diagram showing the in-line setup of the Australian MRI-LINAC is shown in Figure 1, and is described in greater detail in Keall et al. [15] and Liney et al. [16].

Beam profiles were measured using an in-house PSD and Gafchromic EBT3 film (Ashland Inc, USA). These profiles were measured for the smallest symmetric, square fields achievable at SIDs of $1.869 \mathrm{~m}$ and $2.469 \mathrm{~m}$ (being 2 of 8 achievable SIDs for the Australian MRI-LINAC system), where the field sizes were $1.9 \times 1.9 \mathrm{~cm}^{2}$ at $1.869 \mathrm{~m}$ SID and $2.6 \times 2.6 \mathrm{~cm}^{2}$ at $2.469 \mathrm{~m}$ SID. These beam profiles were measured at depths of $1 \mathrm{~cm}, 5 \mathrm{~cm}, 10 \mathrm{~cm}$ and $20 \mathrm{~cm}$ for each SID. A water tank was used to measure the beam profiles with the PSD. The water tank had $8 \mathrm{~mm}$ thick perspex walls with outer dimensions of $37 \times 30$ $\times 42 \mathrm{~cm}^{3}$. A manual linear translation stage (MT-DDA, Med-Tech Inc, USA) with minimum step length of $0.1 \mathrm{~mm}$ was used to control the vertical position of the PSD. Profiles were measured with the PSD by delivering a fixed number of monitor units, recording the PSD's integral response and translating the PSD in $2.5 \mathrm{~mm}$ increments. For each position in each PSD profile, the integral response was measured 3 times, allowing for uncertainties to be calculated. The MRI-LINAC, water tank, translation stage and PSD are shown in Figure 1.

Each measured profile was normalised to its centre of field response, making them relative beam profiles. Each profiles centre of field position was aligned with the origin to minimise differences between corresponding PSD and film profiles. The empirical penumbra model developed by Tang et al. [17 was curve fit to the penumbra in each measured profile. Penumbra widths were calculated by finding the penumbral positions where the relative doses were $20 \%$ and 80 $\%$, and then taking the difference between these $20 \%$ and $80 \%$ penumbral positions. Uncertainties in measured penumbra widths were determined by finding their corresponding $95 \%$ confidence intervals, and adding the uncertainty in each detectors measurement position. For the PSD, the measurement position uncertainty corresponded to half of the translation stage's minimum step length $(0.05 \mathrm{~mm})$; for film, the measurement position uncertainty was half of the pixel width $(0.1 \dot{6} \mathrm{~mm})$.

Global gamma indices $(\gamma)$ and corresponding pass rates were calculated to quantify agreement between the relative PSD profiles and film profiles. The $\gamma_{\mathrm{s}}$ and pass rates were calculated following the protocol outlined by Low et al. [18] for dose/distance criterion of $1 \% / 1 \mathrm{~mm}, 2 \% / 2 \mathrm{~mm}$ and $3 \% / 3 \mathrm{~mm}$ using film profiles as the reference datasets and PSD profiles as the evaluation dataset. No dose thresholds or reference data interpolation techniques were used when calculating $\gamma \mathrm{s}$ and their corresponding pass rates. 


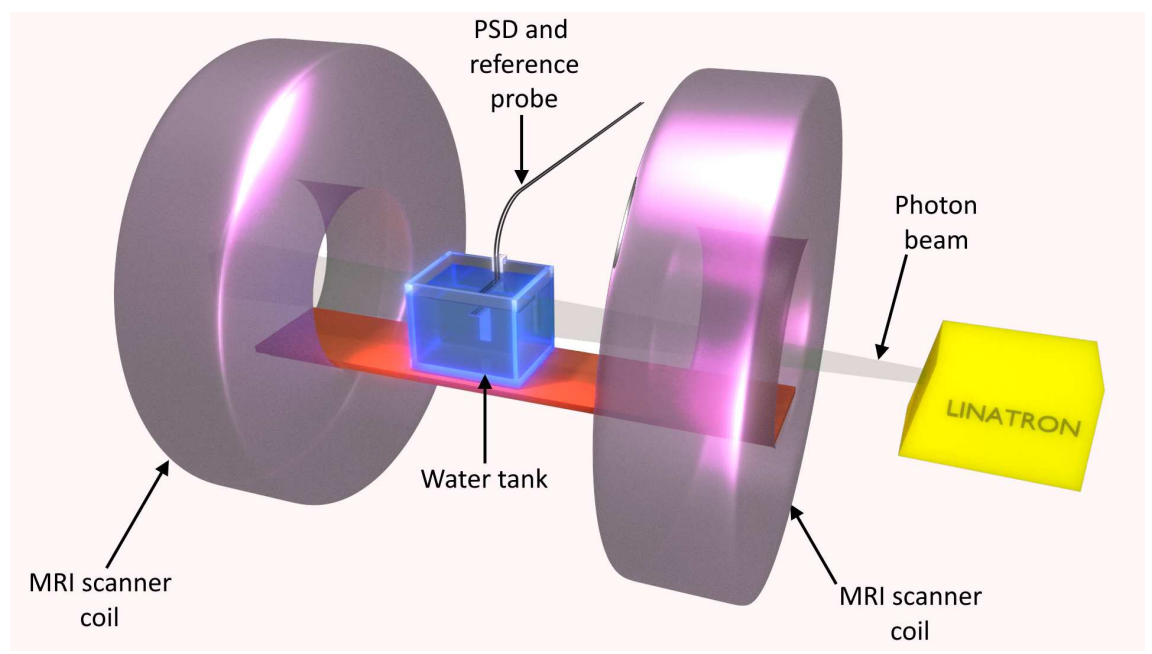

Figure 1: 3D model of the Australian MRI-LINAC with water tank, translation stage, PSD and reference probe.

The Gafchromic EBT3 film was calibrated using an Elekta 6 MV photon beam following the recommendations in AAPM task group report 55 [19]. Solid water (Gammex RMI 457) was used as the phantom material for all measurements with film. For measurement of the beam profiles, the films were placed between the desired thickness of solid water to achieve the desired depth and 10 $\mathrm{cm}$ of solid water for back scatter. Films were scanned using an EPSON V700 Photo flat bed scanner (Epson, Japan), with a resolution of 72 dpi and 48 bit RGB colour depth. Only the red colour channel was used when analysing the films optical density. Each film measured profile was smoothed using a moving average filter with a 5 pixel span.

The PSD consisted of a cylinder of BC444 plastic scintillator (Saint Gobain, France) optically coupled to an Eska CK-40 optical fibre of $15 \mathrm{~m}$ length. The plastic scintillator had a diameter of $2 \mathrm{~mm}$ and a length of $0.8 \mathrm{~mm}$, and the optical fibre had an inner core diameter of $0.94 \mathrm{~mm}$, an outer core diameter of $1 \mathrm{~mm}$ and cladding diameter of $2 \mathrm{~mm}$. A reference probe was constructed from a matching Eska CK-40 optical fibre to that of the PSD. The PSD and reference probe were sealed with black paint to prevent background light from entering the optical fibre. An optical fibre housing was constructed to hold the PSD in the water tank, consisting of a $1 \times 2 \times 16 \mathrm{~cm}^{3}$ piece of solid water with a $2 \mathrm{~mm}$ deep $\times 4 \mathrm{~mm}$ wide groove to house the PSD and reference probe. For background subtraction, the reference probe and PSD were aligned at their tips and placed in the housing groove with the reference probe behind the PSD from the beam's eye view as in Figure 2. This PSD and reference probe alignment ensures that equivalent lengths of optical media are irradiated, and so equivalent magnitudes of Cerenkov are generated in the PSD and reference probe. The optical fibre housing was secured to the manual translation stage, as seen in 


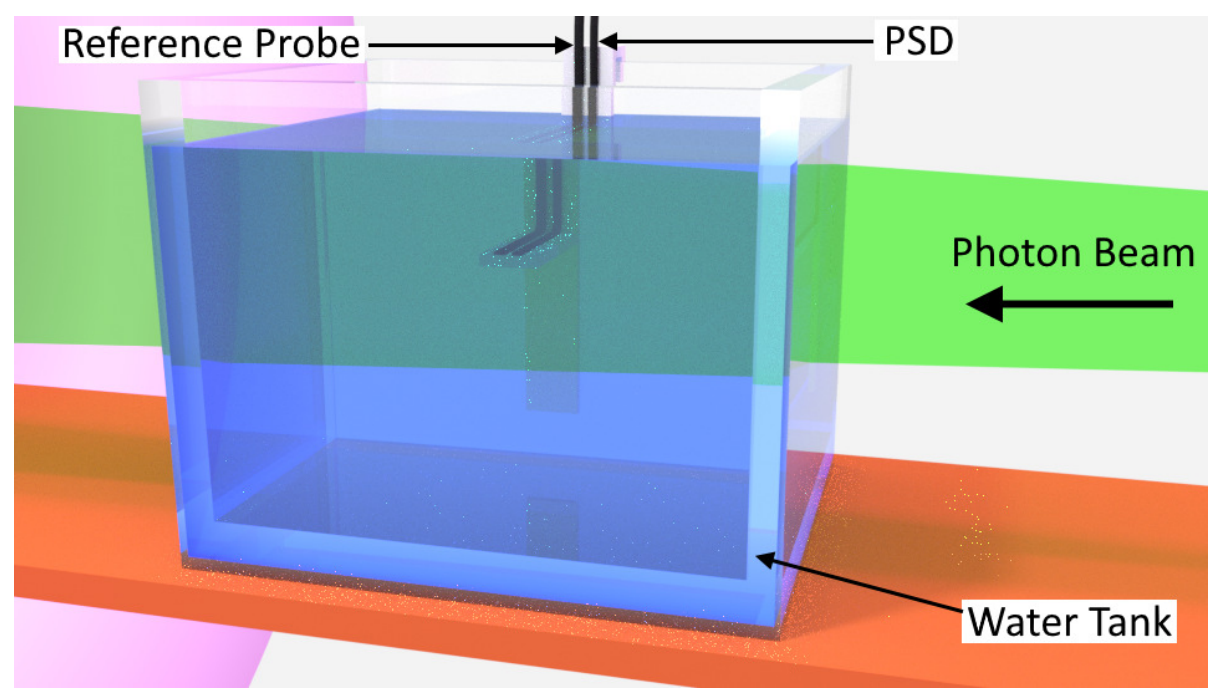

Figure 2: 3D model showing the PSD and reference probe positioning in the water tank. Note that the PSD is placed against the reference probe without a gap between the PSD and reference probe. Beam profiles were measured by changing the vertical position of the PSD in the water tank.

Figure 2. Two matching RCA-4526 photomultiplier tubes (PMTs) and a digital oscilloscope (PicoScope PS6404D, PicoTech) were used to simultaneously measure and record the PSD's and reference probe's optical signals as voltage-time waveforms. The two PMTs were set to AC coupling and the digital oscilloscope sampled the PSD and reference probe waveforms at a rate of $625 \mathrm{MHz}$. The two PMTs were cross calibrated to ensure that the Cerenkov radiation signal measured using one PMT was equivalent to the Cerenkov radiation signal that would be measured using the other PMT.

A scintillating fibre was constructed, such that it would be irradiated by the LINACs beam to produce a triggering signal for the digital oscilloscope as there was no triggering signal available from the LINAC. This scintillating fibre consisted of a $15 \mathrm{~m}$ length of BCF-60 scintillating fibre (Saint Gobain, France) with a core diameter of $1 \mathrm{~mm}$ and a cladding diameter of $2 \mathrm{~mm}$. An aluminium reflector was attached to the end of the scintillating fibre to increase the collected scintillation signal and stop background light from entering the scintillating fibre. The scintillating fibre was secured to the MLC assembly, upstream from the MLC and at a position not intersecting the field shaped by the MLCs. A silicon photomultiplier (SensL MiniSM-30035, Ireland) was used to measure the scintillating fibre's optical signal, with the silicon photomultiplier being connected to the digital oscilloscope for triggering. 


\section{Results}

The beam profiles measured at $1.869 \mathrm{~m}$ SID are shown in Figure 3. Each relative beam profile at $1 \mathrm{~cm}, 5 \mathrm{~cm}, 10 \mathrm{~cm}$ and $20 \mathrm{~cm}$ was scaled by the PSD's relative central axis response for their corresponding depths at $1.869 \mathrm{~m}$ SID (normalised at $5 \mathrm{~cm}$ depth). Linear interpolation was used to sample each film profile at positions where corresponding PSD measurements were made, and relative differences were calculated between corresponding PSD and interpolated film profiles. The film and PSD profiles were within agreement when the calculated relative differences and their corresponding uncertainties intercepted zero. The PSD and film profiles were within agreement for positions within the central $80 \%$ of the beams width (referred to as the central region). The penumbral regions were not in agreement due to the high spatial dose gradient about the penumbral region of the profile. The out of field regions for the PSD and film profiles were generally within agreement, except for the profile at $10 \mathrm{~cm}$ depth about the $-15 \mathrm{~mm}$ position. As this was the only case of disagreement for out of field regions, and was not reproduced in any other profiles, it is expected that this disagreement was a statistical fluctuation. In general, the uncertainty in the relative difference increased as relative dose decreased; this was due to a decrease in the signal to noise ratio as relative dose decreased.

The beam profiles measured at $2.469 \mathrm{~m}$ SID are presented in Figure 4. As in Figure 3, each beam was scaled by the corresponding PSD's relative central axis response for the depths of $1 \mathrm{~cm}, 5 \mathrm{~cm}, 10 \mathrm{~cm}$ and $20 \mathrm{~cm}$ at $2.469 \mathrm{~m} \mathrm{SID;}$ as in Figure 3, the process of scaling by central axis responses was done to minimise profile overlap. The central regions of the PSD and film beam profiles were in good agreement, where the penumbral regions for PSD and film were not in agreement. In the relative difference plot, the out of field regions were in agreement except for the $+22 \mathrm{~mm}$ position. This disagreement is not observed in any other out of field regions and so is attributed to statistical fluctuation.

One dimensional global $\gamma \mathrm{s}$ were calculated for the $1 \% / 1 \mathrm{~mm}, 2 \% / 2 \mathrm{~mm}$ and $3 \% / 3 \mathrm{~mm}$ criterion with no dose threshold. Each beam profile was normalised to its centre of field response, with all profiles being treated as relative beam profiles for $\gamma$ analysis. The calculated $\gamma$ for each criterion are shown in Figure 5. From the calculated $\gamma$ in Figure 5, all the PSD measurements lied within the $3 \% / 3 \mathrm{~mm}$ range, except for the $-8 \mathrm{~mm}$ position for the $1 \mathrm{~cm}$ depth, $1.869 \mathrm{~m}$ SID profile. For the $\gamma$ with $2 \% / 2 \mathrm{~mm}$ criterion, the only PSD positions where $\gamma$ exceeded 1 occurred in the penumbral region, including the aforementioned $-8 \mathrm{~mm}$ position for the $1 \mathrm{~cm}$ depth, $1.869 \mathrm{~m}$ SID profiles. For the $1 \% / 1 \mathrm{~mm}$ criterion, the majority of $\gamma$ exceeding 1 occurred in the penumbral regions. The central regions of the profiles also had a fraction of $\gamma$ exceeding 1, however the distribution of these $\gamma$ exceeding 1 is apparently random and occur due to a combination of spatial variations in the film's response and statistical fluctuations in the PSD's response.

The $\gamma_{\mathrm{s}}$ pass rates are presented in Table 1, with the intention of providing statistics for ranges of agreement between the PSD and film profiles. It should be noted that each PSD profile at $1 \mathrm{~cm}, 5 \mathrm{~cm}$ and $10 \mathrm{~cm}$ depth had 21 measure- 


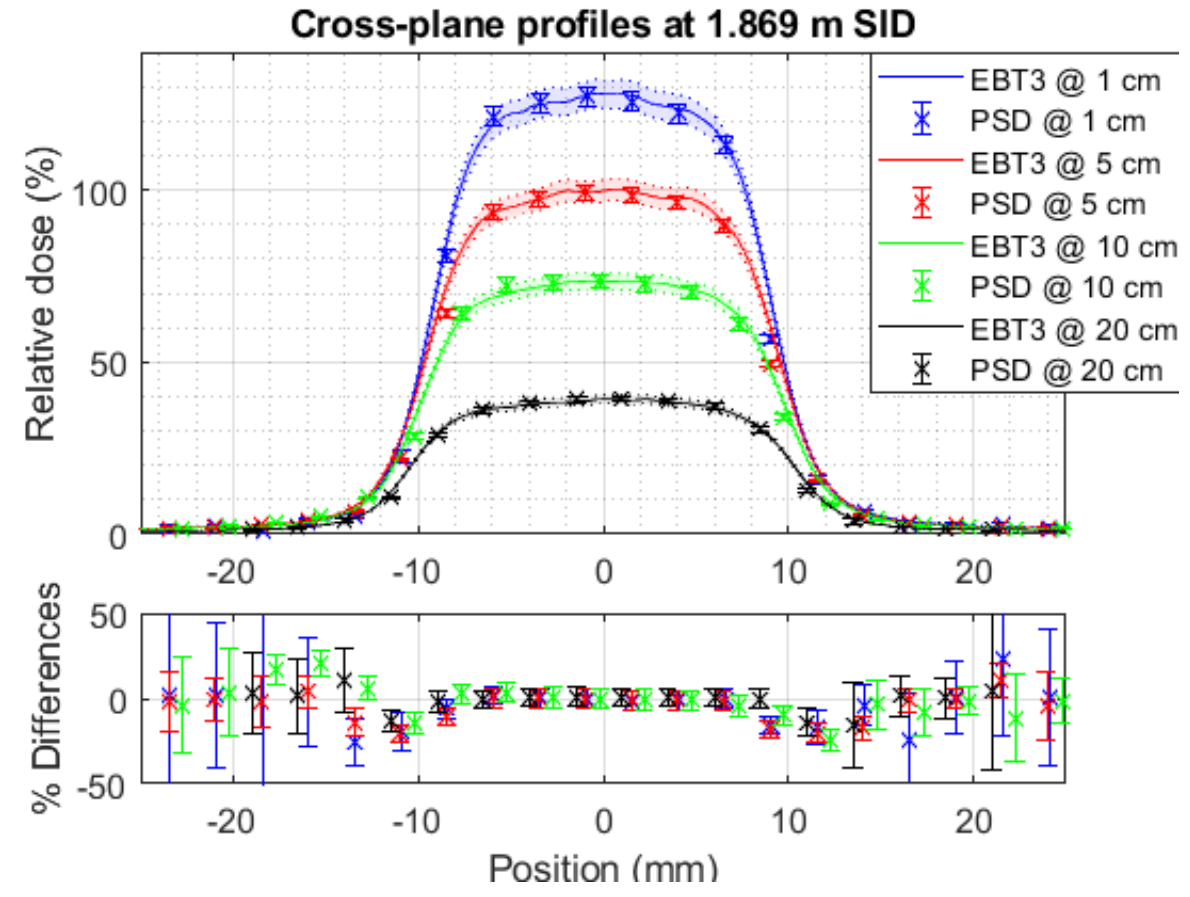

Figure 3: Beam profiles for approximately $1.9 \times 1.9 \mathrm{~cm}^{2}$ field size at isocentre $(10 \mathrm{~cm} \mathrm{depth})$ and $1.869 \mathrm{~m}$ SID (top), and the corresponding relative differences between the PSD and film profiles (bottom). Each PSD and film relative beam profile has been scaled by the PSD's central axis response (normalised to the $5 \mathrm{~cm}$ depth response) at its corresponding depth and field size to minimise the overlap of profiles, however, all beam profiles are treated as relative beam profiles. In the beam profile (top), error bars are twice the combined error in the PSD's measurements, equivalent to the $95 \%$ confidence interval for each measurement and the shaded regions are the uncertainties in the films measurements. In the relative difference plots (bottom), the blue, red, green and black series correspond to the $1 \mathrm{~cm}, 5 \mathrm{~cm}, 10 \mathrm{~cm}$ and $20 \mathrm{~cm}$ depths as in the beam profile plots, respectively. Error bars in the relative difference plots were the combination of the PSD's and film's relative errors.

ment positions, where the $20 \mathrm{~cm}$ depths had 19 measurement positions; a failure in $\gamma$ at each position corresponds to an approximate pass rate reduction of $5 \%$. For the $1 \% / 1 \mathrm{~mm}$ pass rates, pass rated tended to improve as depth increased, however this trend was statistically insignificant. Global pass rates were acceptable for the $2 \% / 2 \mathrm{~mm}$ and $3 \% / 3 \mathrm{~mm}$ criterion, however unacceptable for 1 $\% / 1 \mathrm{~mm}$.

The penumbra widths measured from each relative beam profile are presented in Table 1. Penumbra widths measured at the $1.869 \mathrm{~m}$ SID were on average $(0.2 \pm 0.6) \mathrm{mm}$ narrower when measured with the PSD compared to those measured with film. Similarly, the penumbra widths measured at the $2.469 \mathrm{~m}$ SID were on average $(0.4 \pm 0.8) \mathrm{mm}$ narrower for the PSD compared to those measured with film. The perceived narrowing of penumbras when comparing the PSD with film is not statistically significant per their associated 


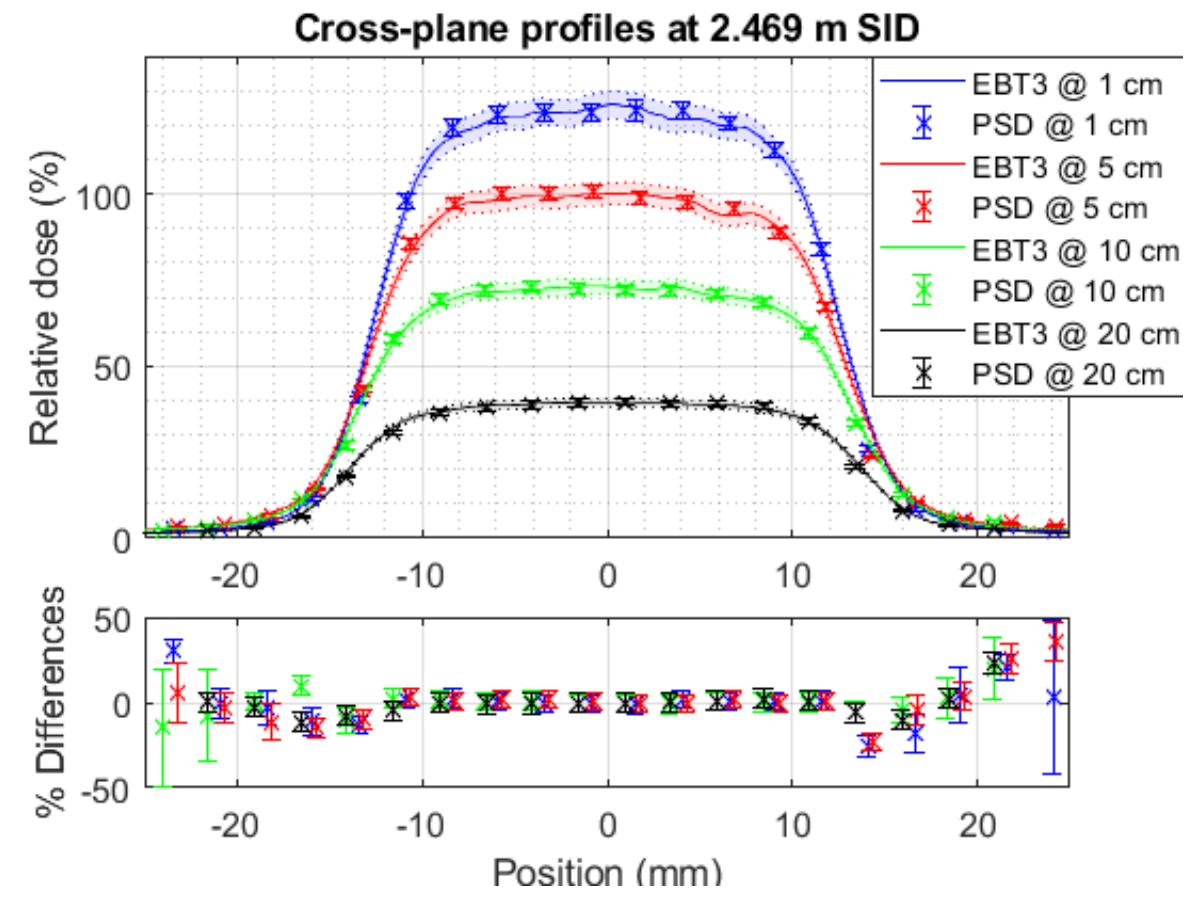

Figure 4: Beam profiles for approximately $2.6 \times 2.6 \mathrm{~cm}^{2}$ field size at isocentre $(10 \mathrm{~cm} \mathrm{depth})$ (top), and relative differences between the PSD and film (bottom). For the EBT3 film and in-house PSD, each relative beam profile was scaled by the PDD value at its corresponding depth, normalised to the centre of field response at $5 \mathrm{~cm}$ depth. Error bars and shaded regions in the beam profile correspond to the uncertainty in the PSD and film, respectively, as in Figure 3. As in Figure 3, the blue, red, green and black series in the relative difference plot corresponds to the $1 \mathrm{~cm}, 5 \mathrm{~cm}, 10 \mathrm{~cm}$ and $20 \mathrm{~cm}$ depths, respectively. Error bars in the relative difference plots were the combination of the PSD's and film's relative errors.

uncertainties. The 5 pixel wide moving average filter used to smooth the film profiles decreased the spatial resolution in the film profiles and widened the film's penumbras. When repeating the analysis with no film smoothing, the resultant film penumbras were on average narrowed by $0.2 \mathrm{~mm}$. Each uncertainty in the film penumbra width varied between cases where smoothing was used and no smoothing was used, however, the global average of the films uncertainty in penumbra width had negligible variance.

\section{Discussion}

In the film profiles, there are spatial response fluctuations in the central regions as can be seen in Figures 3 and 4. These fluctuations are most apparent in the $1 \mathrm{~cm}$ and $5 \mathrm{~cm}$ depth profiles due to the scaling applied, however fluctuations are also present in the $10 \mathrm{~cm}$ and $20 \mathrm{~cm}$ depth profiles in Figures 3 and 4. In a study investigating sources of uncertainty when using EBT3 films, 


\begin{tabular}{|c|c|c|c|c|c|c|c|}
\hline$x$ & $1.869 \mathrm{~m}, 1 \mathrm{~cm}$ & + & $1.869 \mathrm{~m}, 5 \mathrm{~cm}$ & 0 & $1.869 \mathrm{~m}, 10 \mathrm{~cm}$ & $\Delta$ & $1.869 \mathrm{~m}, 20 \mathrm{~cm}$ \\
\hline 米 & $2.469 \mathrm{~m}, 1 \mathrm{~cm}$ & $\square$ & $2.469 \mathrm{~m}, 5 \mathrm{~cm}$ & th & $2.469 \mathrm{~m}, 10 \mathrm{~cm}$ & $\bullet$ & $2.469 \mathrm{~m}, 20 \mathrm{~cm}$ \\
\hline
\end{tabular}

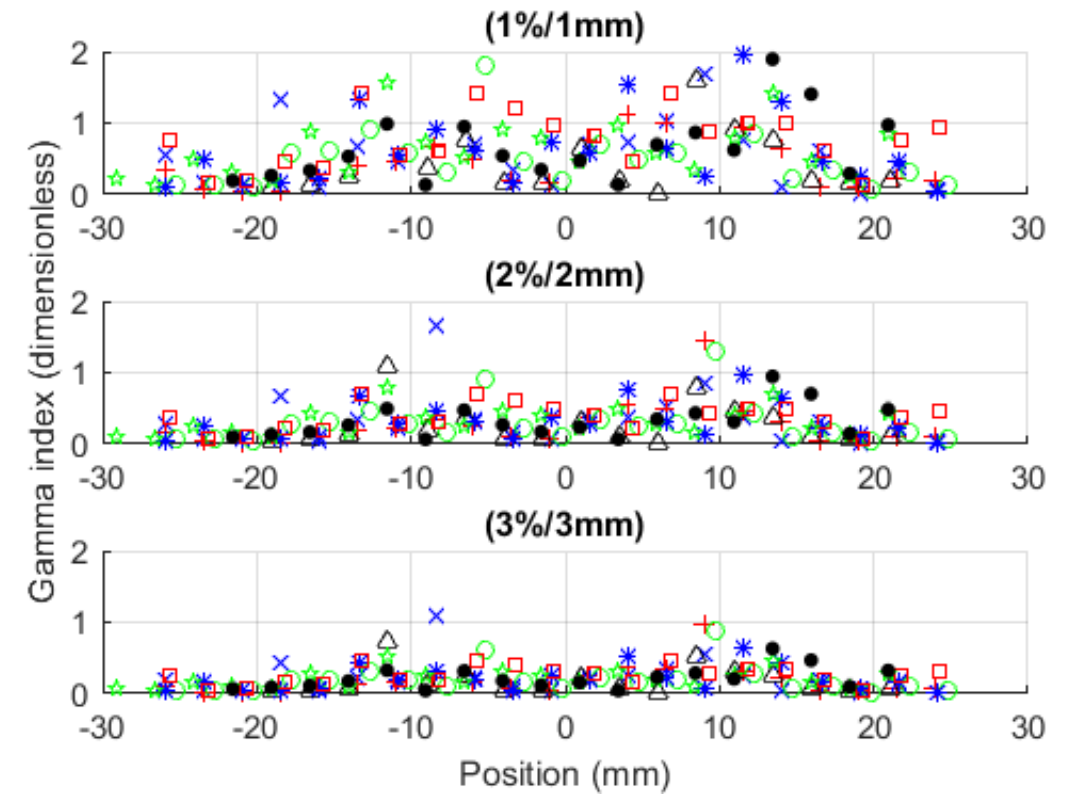

Figure 5: $\gamma \mathrm{s}$ calculated at each position for the $1 \% / 1 \mathrm{~mm}$ criterion (top), $2 \% / 2 \mathrm{~mm}$ criterion (middle) and $3 \% / 3 \mathrm{~mm}$ criterion (bottom) to show any spatial spatial dependences.

measurements of absorbed dose using were found to have a relative uncertainty of $3.2 \%$ when only the red colour channel was used 20 . Film uniformity and reproducibility contributed $0.2 \%$ and $0.2 \%$, respectively; in comparison, the response fitting procedure and dose resolution of the system contributed $2.6 \%$ and $1.8 \%$ 20. In the presented film results, the most significant spatial dose fluctuations occur as under-responses at positions between $+5 \mathrm{~mm}$ and +10 $\mathrm{mm}$. This reproducible under-response is attributed to inhomogeneities in the film, however these fluctuations do not exceed film's $3.2 \%$ relative uncertainty in absorbed dose. Other sources of uncertainty are expected to have other effects; artefacts related to reproducibility would result in random fluctuations, and fitting of responses to dose calibration curves would produce symmetric under-responses.

A potential source of error when using PMTs as the photodetector for PSD measurements can occur as the PMTs undergo temperature changes. A change in PMT temperature causes a change in the PMT's gain; for background subtraction, the ratio of each PMT gains is required. Through their operation, PMTs inevitably produce waste heat and their temperature fluctuates, which can be problematic for the background subtraction method when measurements are taken over a long period of time. Methods of Cerenkov radiation correction that apply a different type of photodetector obviate this potential source of er- 


\begin{tabular}{|c|c|c|c|}
\hline $\begin{array}{c}\text { Profile at } \\
\text { SID, depth }\end{array}$ & $\begin{array}{c}1 \% / 1 \mathrm{~mm} \\
\text { pass rate }\end{array}$ & $\begin{array}{c}2 \% / 2 \mathrm{~mm} \\
\text { pass rate }\end{array}$ & $\begin{array}{c}3 \% / 3 \mathrm{~mm} \\
\text { pass rate }\end{array}$ \\
\hline $1.869 \mathrm{~m}, 1 \mathrm{~cm}$ & $81.0 \%$ & $95.2 \%$ & $95.2 \%$ \\
$1.869 \mathrm{~m}, 5 \mathrm{~cm}$ & $85.7 \%$ & $95.2 \%$ & $100 \%$ \\
$1.869 \mathrm{~m}, 10 \mathrm{~cm}$ & $90.5 \%$ & $95.2 \%$ & $100 \%$ \\
$1.869 \mathrm{~m}, 20 \mathrm{~cm}$ & $88.2 \%$ & $94.1 \%$ & $100 \%$ \\
\hline $2.469 \mathrm{~m}, 1 \mathrm{~cm}$ & $81.0 \%$ & $100 \%$ & $100 \%$ \\
$2.469 \mathrm{~m}, 5 \mathrm{~cm}$ & $81.0 \%$ & $100 \%$ & $100 \%$ \\
$2.469 \mathrm{~m}, 10 \mathrm{~cm}$ & $90.5 \%$ & $100 \%$ & $100 \%$ \\
$2.469 \mathrm{~m}, 20 \mathrm{~cm}$ & $88.9 \%$ & $100 \%$ & $100 \%$ \\
\hline Global mean & $85.8 \%$ & $97.5 \%$ & $99.4 \%$ \\
\hline
\end{tabular}

Table 1: $\gamma$ pass rates calculated using the PSD profiles as the evaluation datasets and film profiles as the reference datasets.

ror; many other research groups use alternative methods of Cerenkov radiation correction, as well as the Exradin W1 and W2 commercial dosimetry systems. Additionally, PMTs with temperature control functionality obviate any problems with changes in gain. In this work the PMT gain drift problem could not be explicitly avoided as the PMTs lacked temperature control functionality; instead, a simple correction method was used. In this approach, several gain calibration measurements were taken throughout the duration of the measurements. Each time of calibration was recorded, as well as each time of profile measurement. The gain drift effects were corrected using linear interpolation to find the time-interpolated gain calibration factors for each time of measurement.

The PSD's sensitive volume was defined by the acceptance light cone of the plastic scintillator (when coupled to the optical fibre). For the setup used (as shown in Figure 2), the effective spatial resolution was the maximum diameter of the acceptance light cone; this was calculated to be $1.8 \mathrm{~mm}$. Film measured penumbra widths were expected to be slightly narrower than PSD penumbra widths as the film's spatial resolution was higher than the PSD's. However, the PSD's measured penumbra widths were on average $0.3 \mathrm{~mm}$ narrower than film's penumbra widths, typically with uncertainties larger than the observed difference in penumbra widths. When the full width at half maximums (FWHMs) were found from the penumbral fitting models, the PSD measured profiles were an average of $(0.6 \pm 0.9) \mathrm{mm}$ narrower than film at $1.869 \mathrm{~m}$ SID and $(0.4 \pm$ $0.8) \mathrm{mm}$ narrower than film at $2.469 \mathrm{~m}$ SID. In general, the uncertainties when comparing the penumbra widths and FWHMs were larger than the combined uncertainties in these differences, bringing these quantities into agreement. For the PSD and film, the uncertainty in each measurement position was $0.05 \mathrm{~mm}$ and $0.1 \dot{6} \mathrm{~mm}$, respectively, arising from the $0.1 \mathrm{~mm}$ step size of the translation stage and $0 . \dot{3} \mathrm{~mm}$ pixel size when scanning film. The other contribution to uncertainties arose with the standard error from the fitted models when measuring 


\begin{tabular}{|c|c|c|c|c|}
\hline \multirow{2}{*}{$\begin{array}{c}\text { Profile at } \\
\text { SID, depth }\end{array}$} & \multicolumn{2}{|c|}{ Left penumbra $(\mathrm{mm})$} & \multicolumn{2}{c|}{ Right penumbra $(\mathrm{mm})$} \\
\cline { 2 - 5 } & Film & PSD & Film & PSD \\
\hline $1.869 \mathrm{~m}, 1 \mathrm{~cm}$ & $3.4 \pm 0.4$ & $3.0 \pm 0.1$ & $3.5 \pm 0.2$ & $3.1 \pm 0.1$ \\
$1.869 \mathrm{~m}, 5 \mathrm{~cm}$ & $3.6 \pm 0.3$ & $3.5 \pm 0.1$ & $3.6 \pm 1.0$ & $3.4 \pm 0.1$ \\
$1.869 \mathrm{~m}, 10 \mathrm{~cm}$ & $3.7 \pm 0.3$ & $3.2 \pm 0.8$ & $3.8 \pm 0.3$ & $3.7 \pm 0.1$ \\
$1.869 \mathrm{~m}, 20 \mathrm{~cm}$ & $3.8 \pm 0.3$ & $3.7 \pm 0.1$ & $3.9 \pm 0.2$ & $3.6 \pm 0.2$ \\
\hline $2.469 \mathrm{~m}, 1 \mathrm{~cm}$ & $4.0 \pm 0.4$ & $3.4 \pm 0.2$ & $4.2 \pm 0.4$ & $3.5 \pm 0.2$ \\
$2.469 \mathrm{~m}, 5 \mathrm{~cm}$ & $4.2 \pm 0.4$ & $3.6 \pm 0.1$ & $4.6 \pm 0.3$ & $4.0 \pm 0.2$ \\
$2.469 \mathrm{~m}, 10 \mathrm{~cm}$ & $4.4 \pm 0.3$ & $4.1 \pm 0.4$ & $4.6 \pm 0.9$ & $4.4 \pm 0.3$ \\
$2.469 \mathrm{~m}, 20 \mathrm{~cm}$ & $4.6 \pm 0.7$ & $4.7 \pm 0.1$ & $4.6 \pm 0.5$ & $4.2 \pm 0.7$ \\
\hline
\end{tabular}

Table 2: Penumbra widths measured from PSD and film profiles. Uncertainties are a combination of the uncertainty in each detectors measurement position $(0.05 \mathrm{~mm}$ for the PSD and $0.16 \mathrm{~mm}$ for the film), and the standard error in the penumbra width (calculated by using the standard error of regression from the curve fitting process to find the $95 \%$ confidence interval in the penumbra widths.)

penumbra width and FWHM. The perceived differences between the PSD and film penumbra widths were statistically insignificant considering the uncertainties in these differences.

With regards to the PSD used in this work, the cylindrical sensitive volume had a diameter of $2 \mathrm{~mm}$, a length of $0.8 \mathrm{~mm}$ and a density of $1.032 \mathrm{~g} / \mathrm{cm}^{3}$. The PSD's small sensitive volume size and density close to water make the effective point of measurement (EPOM) of the PSD the centre of the scintillator cylinder (for no magnetic field). A detector's EPOM shifts in the presence of strong magnetic fields. This shift is dependent on magnetic field strength [21, MRI-LINAC orientation [22], a detector's sensitive volume density [21] and any casing around the sensitive volume [3. From a Monte Carlo study by Looe et al. 21], detectors with sensitive volume densities matching water were found to have their EPOM shift match the shift in absorbed dose distributions deposited in water, irrespective of magnetic field strength. With regards to MRI-LINAC orientation, EPOM shifts laterally and depth-wise for perpendicular orientations [3], but EPOM shifts were limited to depth-wise shifts, and magnitudes of EPOM shift were significantly reduced for in-line orientations [22]. With the considerations that the PSD's sensitive volume's size is small, its density is close to water and the Australian MRI-LINAC's in-line orientation, the shift in EPOM was expected to be of negligible effect for the setup used. For PSDs with small sensitive volumes, it is expected that the EPOM shift would also be practically negligible for other PSDs for MRI-LINACs with in-line orientations. For perpendicular MRI-LINAC orientations, it is expected that the EPOM shift would be negligible due to the typically small volumes of PSDs and their near-water densities.

In the $\gamma$ plots in Figure 5, many of the observed $\gamma$ exceeding 1 occurred in penumbra regions of the beam profiles. Film penumbra widths ranged between 
$3.4 \mathrm{~mm}$ and $4.5 \mathrm{~mm}$; in the penumbral region, this corresponds to a minimum change of $4.6 \%$ relative dose per pixel. This makes some PSD penumbral measurements fail, even though some of these measurements appear to overlap with corresponding film profiles. For the $2 \% / 2 \mathrm{~mm}$ and $3 \% / 3 \mathrm{~mm}$ criterion, the positions with $\gamma$ exceeding 1 would likely pass if the scanning resolution were higher. For the $1 \% / 1 \mathrm{~mm}$ criterion, there are $\gamma$ exceeding 1 in the central and penumbral regions. Improvements could be expected for the $1 \% / 1 \mathrm{~mm}$ criterion penumbral regions if a higher scanning resolution was used. However, with the observed, yet statistically insignificant differences in penumbra widths and FWHM, the penumbral regions would likely still have some $\gamma$ exceeding 1 in the penumbral regions at the $1 \% / 1 \mathrm{~mm}$ tolerance.

Many other dosimeters have been investigated for their efficacy in beam profile measurements. Chen et al. verified an MRI-LINAC's treatment planning system (TPS) using a micro-ionisation chamber, a Farmer-ionisation chambers, Gafchromic EBT3 film and a PTW60019 microDiamond detector 23. For beam profiles, these detectors were found to be within a $1 \%$ global agreement with the TPS for out of field regions, and $2 \%$ global agreement for the central regions 23. For the same conditions, when calculating dose differences between the PSD and film profiles as relative beam profiles, the PSD and film were found to be within $2.2 \%$ global agreement for the central regions and $1.5 \%$ global agreement for out of field regions. These statistics suggest that the in-house PSD's performance is similar to many other commercially available dosimeters used to measure MRI-LINAC beam profiles.

The work presented in this manuscript has demonstrated that the in-house PSD's performance was comparable to other commercially available dosimeters. However, as only one in-house PSD was used in this work, the translation of these results to other PSDs may be problematic as there exists plastic scintillator materials that do not possess all aforementioned desirable properties for dosimetry. For example, studies have shown that their PSDs investigated had negligible temperature dependences [8, 10; however, other scintillator materials have been shown to have non-negligible temperature dependences [24, 25]. In previous work, an in-house PSD using BC444 (Saint Gobain, France) as the scintillator material, was used to measure output factors at the Australian MRILINAC. No field size dependences were observed for the field sizes ranging between $2.6 \times 2.6 \mathrm{~cm}^{2}$ and $21.0 \times 21.0 \mathrm{~cm}^{2}$ 14; however, Exradin W1 (Standard Imaging, USA ) exhibited field size dependences in the presence of a magnetic field when field sizes were greater than $10.5 \times 10.5 \mathrm{~cm}^{2}$ [26]. With other PSD's desirable properties verified, these results can be expected to translate to other PSDs.

\section{Conclusion}

An in-house PSD and Gafchromic EBT3 film were applied to measure beam profiles with an in-line MRI-LINAC. Mean global $\gamma$ pass rates were calculated using the film as a reference for the PSD. Pass rates were $85.8 \%, 97.5 \%$ and $99.4 \%$ for the $1 \% / 1 \mathrm{~mm}, 2 \% / 2 \mathrm{~mm}$ and $3 \% / 3 \mathrm{~mm}$ criteria, respectively. The 
majority of the $\gamma$ failures occurred in the penumbral regions, which were partially exacerbated by the 72 dpi spatial sampling during film scanning. Penumbra widths were measured to be slightly narrower with the PSD compared to film, however, their uncertainties made the narrowing statistically insignificant. Differences in dose between the PSD and film remained within $2.2 \%$ global agreement for the central regions and $1.5 \%$ global agreement for out of field regions. These statistics for difference in dose are similar to the those reported in the literature for other dosimeters that are trusted for MRI-LINAC dosimetry.

\section{Acknowledgements}

The Australian MRI-linac program is supported by the Australian NHMRC Research Council Program Grant APP1132471. This research has been conducted with the support of the Australian Government Research Training Program Scholarship. This research has been conducted with scholarship support from the South West Sydney Local Health District.

\section{References}

[1] Godley A, Zheng D, Rong Y. Mr-linac is the best modality for lung sbrt. Journal of Applied Clinical Medical Physics 2019;20(6):7-11. doi 10.1002/ acm2.12615.

[2] Corradini S, Alongi F, Andratschke N, Belka C, Boldrini L, Cellini F, et al. Mr-guidance in clinical reality: current treatment challenges and future perspectives. Radiation Oncology 2019;14:92. doi 10.1186/ s13014-019-1308-y.

[3] O'Brien DJ, Dolan J, Pencea S, Schupp N, Sawakuchi GO. Relative dosimetry with an mr-linac: Response of ion chambers, diamond, and diode detectors for off-axis, depth dose, and output factor measurements. Medical Physics 2018;45(2):884-897. doi 10.1002/mp.12699.

[4] Barten DLJ, Hoffmans D, Palacios MA, Heukelom S, van Battum LJ. Suitability of EBT3 GafChromic film for quality assurance in MR-guided radiotherapy at $0.35 \mathrm{t}$ with and without real-time MR imaging. Physics in Medicine \& Biology 2018;63(16):165014. doi:10.1088/1361-6560/aad58d.

[5] Delfs B, Schoenfeld AA, Poppinga D, Kapsch RP, Jiang P, Harder D, et al. Magnetic fields are causing small, but significant changes of the radiochromic EBT3 film response to $6 \mathrm{MV}$ photons. Physics in Medicine \& Biology 2018;63(3):035028. doi 10.1088/1361-6560/aa9bd5.

[6] Woodings SJ, Wolthaus JWH, van Asselen B, de Vries JHW, Kok JGM, Lagendijk JJW, et al. Performance of a PTW 60019 microDiamond detector in a 1.5 t MRI-linac. Physics in Medicine \& Biology 2018;63(5):05NT04. doi:10.1088/1361-6560/aaa1c6. 
[7] Wegener S, Weick S, Sauer OA. Influence of a transverse magnetic field on the response of different detectors in a high energy photon beam near the surface. Zeitschrift für Medizinische Physik 2019;29(1):22 - 30. doi 10 . 1016/j.zemedi.2018.07.001.

[8] Beddar AS, Mackie TR, Attix FH. Water-equivalent plastic scintillation detectors for high-energy beam dosimetry: II. properties and measurements. Physics in Medicine and Biology 1992;37(10):1901-1913. doi 10. 1088/0031-9155/37/10/007.

[9] Clift MA, Sutton RA, Webb DV. Water equivalence of plastic organic scintillators in megavoltage radiotherapy bremsstrahlung beams. Physics in Medicine and Biology 2000;45(7):1885-1895. doi 10.1088/0031-9155/ $45 / 7 / 313$.

[10] Dimitriadis A, Patallo IS, Billas I, Duane S, Nisbet A, Clark C. Characterisation of a plastic scintillation detector to be used in a multicentre stereotactic radiosurgery dosimetry audit. Radiation Physics and Chemistry 2017;140:373 - 378. doi $10.1016 / j . r a d p h y s c h e m .2017 .02 .023$.

[11] Therriault-Proulx F, Wen Z, Ibbott G, Beddar S. Effect of magnetic field strength on plastic scintillation detector response. Radiation Measurements 2018;116:10 - 13. doi $10.1016 /$ j.radmeas. 2018.06.011.

[12] Beaulieu L, Beddar S. Review of plastic and liquid scintillation dosimetry for photon, electron, and proton therapy. Physics in Medicine and Biology 2016;61(20):R305-R343. doi:10.1088/0031-9155/61/20/R305.

[13] Hirano Y, Yamamoto S. Angular dependencies of cerenkov-light in water for carbon-ion, high energy x-ray and electron. Biomedical Physics \& Engineering Express 2019;5(2):027003. doi 10.1088/2057-1976/ab05b0.

[14] Madden L, Archer J, Li E, Jelen U, Dong B, Roberts N, et al. First measurements with a plastic scintillation dosimeter at the australian MRILINAC. Physics in Medicine \& Biology 2019;64(17):175015. doi 10.1088/ 1361-6560/ab324b.

[15] Keall PJ, Barton M, Crozier S. The australian magnetic resonance imaging-linac program. Seminars in Radiation Oncology 2014;24(3):203 - 206. doi $10.1016 / j . s e m r a d o n c .2014 .02 .015$ magnetic Resonance Imaging in Radiation Oncology.

[16] Liney GP, Dong B, Begg J, Vial P, Zhang K, Lee F, et al. Technical note: Experimental results from a prototype high-field inline mri-linac. Medical Physics 2016;43(9):5188-5194. doi:10.1118/1.4961395.

[17] Tang SQ, Jen YM, Wu JM. An empirical model for describing the small field penumbra in radiation therapy. BioMed Research International 2019;2019:1-13. doi:10.1155/2019/7584743. 
[18] Low DA, Harms WB, Mutic S, Purdy JA. A technique for the quantitative evaluation of dose distributions. Medical Physics 1998;25(5):656-661. doi $10.1118 / 1.598248$.

[19] Niroomand-Rad A, Blackwell CR, Coursey BM, Gall KP, Galvin JM, McLaughlin WL, et al. Radiochromic film dosimetry: Recommendations of aapm radiation therapy committee task group 55. Medical Physics 1998;25(11):2093-2115. doi 10.1118/1.598407.

[20] Marroquín EYL, González JAH, López MAC, Barajas JEV, GarcíaGarduño OA. Evaluation of the uncertainty in an ebt3 film dosimetry system utilizing net optical density. In: Journal of applied clinical medical physics. 2016,doi:10.1120/jacmp.v17i5.6262.

[21] Looe HK, Delfs B, Poppinga D, Harder D, Poppe B. Magnetic field influences on the lateral dose response functions of photon-beam detectors: MC study of wall-less water-filled detectors with various densities. Physics in Medicine and Biology 2017;62(12):5131-5148. doi 10.1088/1361-6560/ aa6ca0.

[22] Gargett M, Oborn B, Metcalfe P, Rosenfeld A. Monte carlo simulation of the dose response of a novel $2 \mathrm{~d}$ silicon diode array for use in hybrid mri-linac systems. Medical Physics 2015;42(2):856-865. doi:10.1118/1.4905108.

[23] Chen X, Paulson E, Ahunbay E, Sanli A, Klawikowski S, Li X. Measurement validation of treatment planning for a mr-linac. Journal of Applied Clinical Medical Physics 2019;20(7):28-38. doi:10.1002/acm2.12651.

[24] Buranurak S, Andersen C, Beierholm A, Lindvold L. Temperature variations as a source of uncertainty in medical fiber-coupled organic plastic scintillator dosimetry. Radiation Measurements 2013;56:307 - 311. doi $10.1016 / j . r a d m e a s .2013 .01 .049$ proceedings of the 8th International Conference on Luminescent Detectors and Transformers of Ionizing Radiation (LUMDETR 2012).

[25] Wootton L, Beddar S. Temperature dependence of BCF plastic scintillation detectors. Physics in Medicine and Biology 2013;58(9):2955-2967. doi 10 . 1088/0031-9155/58/9/2955

[26] Yoon J, Kim JI, Choi CH, Park JM. Characteristics of the exradin w1 scintillator in the magnetic field. Journal of applied clinical medical physics 2019;20(9):149 - 156. doi:10.1002/acm2.12707. 